\title{
Body Image in Athletes and Nonathletes With Low Back Pain: Avoidance-Endurance-Related Subgroups and Sports Status Play a Role
}

\author{
Claudia G. Levenig, Michael Kellmann, Jens Kleinert, Johanna Belz, Tobias Hesselmann, \\ Jahan Heidari, and Monika I. Hasenbring
}

\begin{abstract}
Context: Low back pain (LBP) is a serious health problem, both in the general population as well as in athletes. Research has shown that psychosocial aspects, such as dysfunctional pain responses, play a significant role in the chronification of LBP. Recent research supports the relevance of the multidisciplinary concept of body image in the interpretation of LBP. Objective: To examine the differences in 2 psychosocial aspects, body image and pain responses, between athletes and nonathletes with LBP. Design: Cross-sectional design. Setting: The questionnaires were distributed in the course of LBP treatment. Participants: Data from 163 athletes (mean age $=28.69$ [9.6] y) and 75 nonathletes (mean age $=39.34$ [12.63] y) were collected. Interventions: Data were collected by questionnaires assessing body image, pain behavior, training activity, and LBP. Main Outcome Measures: To examine group differences between athletes and nonathletes regarding body image and pain behavior, the authors performed 2-way analyses of variance with Bonferroni post hoc tests. Results: The results showed (1) a significant main effect regarding pain responses and body image, showing that participants with eustress endurance or adaptive pain behavior revealed a more positive body image in both groups compared with participants with distress endurance or fear-avoidance behavior, and (2) a significant main effect for the factor group in the body image dimension of physical efficacy, indicating a more positive body image for athletes. Conclusion: These results suggest that considering multiple risk factors for LBP, such as body image and dysfunctional pain behavior, as well as subgrouping, might be valuable for research and for broadening therapy options.
\end{abstract}

Keywords: pain response, pain behavior, physical activity, cognitive-affective aspects of body

Low back pain (LBP) is one of the most common health problems in the general population, with lifetime prevalence rates reported as high as $80 \%{ }^{1}$ These prevalence rates are largely applicable for high-performance athletes, as well. ${ }^{2}$ Although psychosocial aspects are well examined and accepted as risk factors for maintenance for the general population, ${ }^{1,3}$ LBP in athletes primarily is discussed with respect to biomechanical risks and causes. ${ }^{4}$ Psychosocial risk factors particularly involve emotional distress and heightened depressive mood, as well as dysfunctional responses to pain, including cognitive, emotional, and behavioral characteristics, often followed by not feeling able to participate in a social life. ${ }^{5}$ This line of research stimulated the concept of subgrouping the heterogeneous population of patients with LBP with respect to their psychosocial risk of chronification and allowed for the integration of individually tailored approaches to pain treatment. ${ }^{3,5}$

Hasenbring et $\mathrm{al}^{5}$ have shown that several subgroups can be distinguished among patients with LBP. A high risk of pain chronification is assumed due to either elevated pain-related fear

Levenig and Hasenbring are with the Department of Medical Psychology and Medical Sociology; Kellmann, Hesselmann, and Heidari are with the Unit of Sport Psychology, Faculty of Sport Science; Ruhr University Bochum, Bochum, Germany. Kellmann is also with the School of Human Movement and Nutrition Sciences, The University of Queensland, Brisbane, QLD, Australia. Kleinert and Belz are with the Department of Health and Social Psychology; Kleinert is also with the German Research Center of Competitive Sport; German Sport University Cologne, Cologne, North Rhine-Westphalia, Germany. Levenig (levenig@ medpsych.ruhr-uni-bochum.de) is corresponding author. avoidance or dysfunctional endurance pain responses, while in contrast, an adaptive group yields a low risk for chronic pain and disability. Possible mechanisms that may foster a process of pain chronification are described within the Avoidance-Endurance Model (AEM) of pain. 6,7 The AEM (see Figure 1) postulates 3 dysfunctional and 1 functional response patterns to pain. First, the fear-avoidance response (FAR) is specified by catastrophizing thoughts (eg, "It is not serious, is it?"5), resulting from an overinterpretation of the threat of pain, leading to fear, depressive mood, distinctive avoidance behavior (eg, avoiding visiting friends ${ }^{5}$ ), and physical inactivity. ${ }^{8,9}$ Furthermore, 2 patterns describe endurancerelated pain responses: patients may tend to show so-called distress-endurance responses (DERs), comprising thought suppression as a cognitive response to pain (eg, "Don't think about your pain."5) in order to not feel the pain. There is ample evidence that thought suppression is often ineffective in reducing pain, with increased emotional distress and depressive mood as emotional consequences. ${ }^{5,10}$ Patients may further respond with an eustressendurance response (EER) pattern, characterized by cognitions of focused distraction from pain (eg, concentrating on valuable effects of an activity $)^{5}$ and the ability to maintain a positive mood despite pain. Both endurance patterns are characterized by physical overactivity behavior and, therefore, overload of physical structures, such as muscles, discs, or fascia. Finally, the AEM assumes an adaptive response (AR) to pain, describing patients' ability to cope with pain in a more functional way (ie, finding a balance between load and recovery). ${ }^{6,7,11}$ Athletes often show high levels of endurance behavior, which is evoked by an enormous pressure. This pressure is evoked by trainers, media, and audience who expect top 


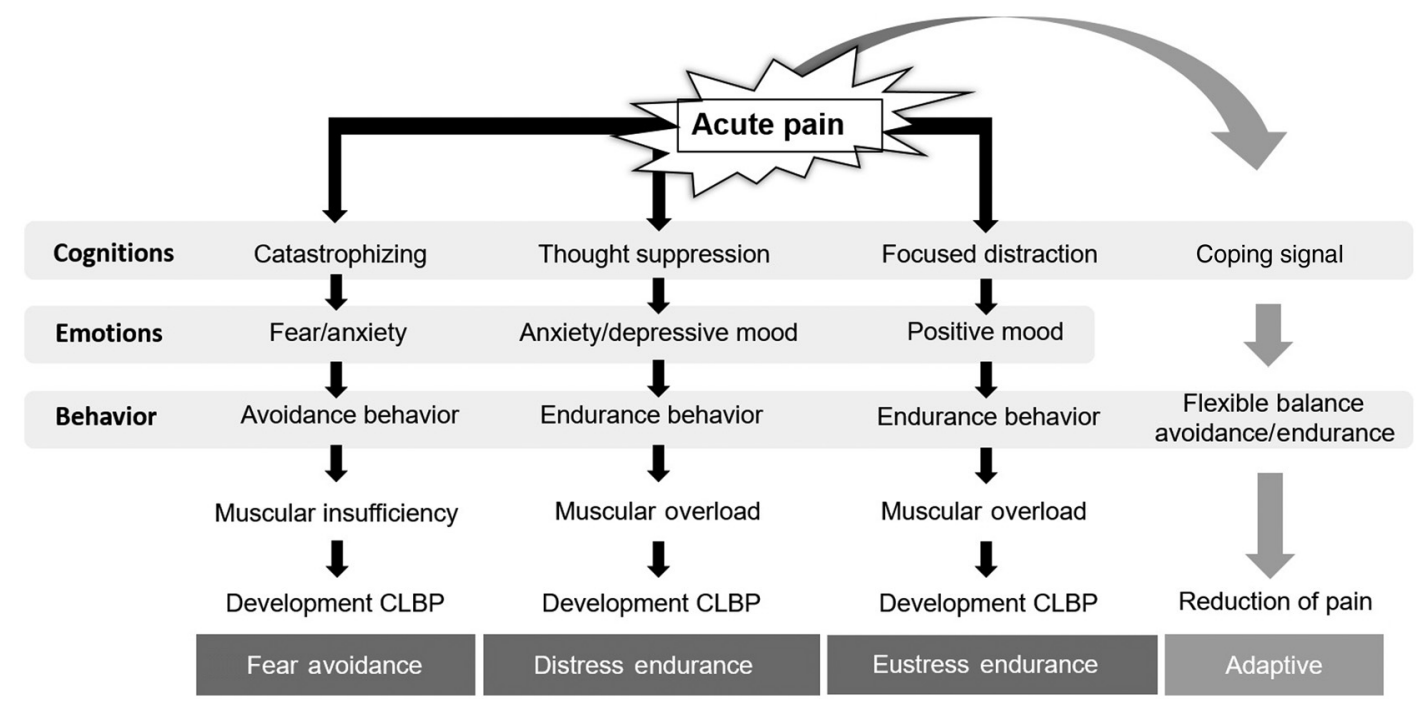

Figure 1 - The Avoidance-Endurance Model of pain.

performance independent from injury or pain. ${ }^{12}$ Preliminary research indicates that endurance-related pain responses apart from FAR are also associated with higher pain and disability in athletes with $\mathrm{LBP}^{13}$; however, little is known about the role of psychosocial subgroups in athletes.

During the past decade, unfavorable facets of body image became of increasing interest, that is, perceptual aspects, such as misperceptions of the painful body part (eg, "I can't find it," "It feels as though it has shrunk" 14 ), and cognitive-affective aspects of body image, the latter referring to negative evaluations of one's own body with respect to self-acceptance (eg, "I have more physical deficits than others"15), health (eg, "I often reach my physical limits" ${ }^{15}$ ), and physical efficacy (eg, "I am strong"15). ${ }^{14-17}$ Recently, it has been shown that nonathletic patients with LBP reveal a more negative body image than healthy controls, although the direction of this relationship remains open. ${ }^{17}$ However, there are inconsistent results regarding athletes. On the one hand, physical activity has been shown to evoke a more positive body image ${ }^{18}$ Furthermore, athletes experience respect of the audience, trainer, and the media if they fulfill the expectations and show top performances. In particular, athletes usually represent a societal body ideal of a functional and well-trained body. ${ }^{19}$ On the other hand, in athletes, the experienced pressure to endure pain is suggested to lead to a negative body image, because pain is normalized, suppressed, or trivialized. ${ }^{12}$ Moreover, some studies have shown that athletes are dissatisfied with their body image because of certain demands that are sport immanent, like weight control in high jumpers, ski jumpers, or dancers. ${ }^{20-23}$ It can therefore be argued that high levels of endurance, shown in response to pain, may lead to a more negative body image. ${ }^{24,25}$ This might be true at least in subgroups of individuals suffering from LBP, who display a DER pattern according to the AEM. A negative body image, however, might also be present in individuals showing FAR, as damage to the body could lead to anticipatory anxiety and negative thoughts. ${ }^{26}$ However, athletes showed lower levels of avoidance compared with nonathletes. ${ }^{27}$

This study aimed to increase knowledge about possible distortions in body image in athletes versus nonathletes with LBP and about the role of psychosocial subgroup differences, which might serve to improve the process of individualization in multimodal pain treatment approaches. The consideration of psychosocial factors that may contribute to the maintenance of clinical pain syndromes is needed, especially in the training and treatment of athletes with LBP, because most programs for athletes are merely oriented in an improvement of performance. Concepts that adequately address clinical pain syndromes are lacking.

To the best of our knowledge, this is the first study to address body image in athletes and nonathletes, both suffering from LBP, with respect to psychosocial subgroup differences. We hypothesized that subgroups showing a dysfunctional pattern of pain responses, either fear-avoidance or endurance pattern (especially the DER pattern), tend to show a more negative body image, irrespective of their training status. We further hypothesized that, even in the case of LBP and social pressure, athletes display a more positive body image than nonathletes, justified by lower levels of avoidance.

\section{Methods}

\section{Participants and Design}

This present study is part of a larger cross-sectional multicenter study that examined the psychosocial aspects of LBP in athletes and nonathletes. ${ }^{28}$ The inclusion criteria for all participants in the present study were the presence of nonspecific LBP, an age between 18 and 70 years, and participation in a prescribed exercise treatment or therapy. The exclusion criteria for both, athletes and nonathletes, were medical conditions like acute fractures, infections, tumors, rheumatic or other chronic pain diseases, and known psychiatric disorders, like psychoses.

The participants who self-reported to be a competitive athlete at an international, national, or regional level and indicated a weekly training volume of at least 3 hours were allocated to the athlete group. The participants who were active on a local or noncompetitive level were assigned to the nonathlete group. This categorization is based on recommendations by Araújo and Scharhag $^{29}$ to define athletes by their performance level and a second criterion like training volume. 
In the group of nonathletes, a total of 167 participants were screened. Of the 167 participants, 31 declined to participate and 40 were excluded for not fulfilling the inclusion criteria (4 due to missing LBP, 3 for not fulfilling the age inclusion criterion, and 33 because of exclusion criteria). Of the remaining 96 participants, 12 were excluded due to incomplete data. Finally, the data of 84 participants were included. Nine of them were categorized as athletes because they revealed a high-training frequency and a higher competitive level, fulfilling the criteria for athletes. ${ }^{29}$ Finally, the group of nonathletes consisted of 75 participants. One-hundred seventy-nine athletes were recruited; 16 were excluded, following the exclusion criteria. The group of athletes finally consisted of 163 participants. The total sum of participants was 238 .

\section{Procedure}

Individuals from the general population were recruited from rehabilitation and health institutions and fitness centers in North Rhine-Westphalia, Germany. Athletes were recruited via physiotherapists of various German Olympic training centers. After the recruitment, data were checked for the participants' indicated performance level and weekly training activity. All participants received written details about the content of the study and a gift certificate of $10 €$. All participants gave their written consent. The medical ethics committee of Ruhr University Bochum approved the study protocol.

\section{Materials}

Demographic Variables. The participants' demographic and physical training variables were assessed by questionnaires.

Pain Outcomes. The participant's pain history was assessed with a short form of the German version of the Chronic Pain Grade. ${ }^{30,31}$ The participants indicated their current, average, and highest pain intensity within the previous 3 months on a 11-point Likert scale ranging from 0 ("no pain") to 10 ("pain as bad as could be"). The Cronbach alpha was .68 for the sum score. Furthermore, they specified their pain-related disability of daily, leisure, and work activities (Cronbach $\alpha=.88$ ) on an 11-point Likert scale ranging from 0 ("no interference") to 10 ("unable to carry on my activities"). All participants were classified as suffering from subacute LBP if their pain duration was $<90$ days and as suffering from chronic LBP if their pain duration was $\geq 90$ days. $^{32}$

Frankfurt Body Concept Scales. The cognitive-affective aspects of body image were assessed using the Frankfurt Body Concept Scales (German: Frankfurter Körperkonzeptskalen ${ }^{15}$ ), a self-rating instrument, which consists of 9 subscales assessing aspects of the individual body concept. Three subscales were chosen in the present study: health (6 items, Cronbach $\alpha=.81$ ), physical efficacy (10 items, Cronbach $\alpha=.90$ ), and self-acceptance of one's body (6 items, Cronbach $\alpha=.81$ ). Health comprises individual attitudes based on experiences like malfunction or function of the body and feeling healthy or unhealthy. Physical efficacy is described as individual attitudes toward aspects such as strength and flexibility. Self-acceptance is characterized as evaluations about the past and present life, as well as attractiveness. ${ }^{15}$ All items were rated by a 6-point Likert scale ranging from "very much" to "not at all." The higher the score, the more positive is the body image. A positive body image can be assumed with scores $>24$ on health and selfacceptance, and $>40$ on physical efficacy. Positive correlations for the 3 body image dimensions between .49 and .56 show overlapping but also distinct features.

Pain Responses. The German version of the AvoidanceEndurance Questionnaire (AEQ) ${ }^{33}$ is a self-report instrument assessing both FAR and endurance responses to pain. The AEQ consists of 9 subscales. The AEQ has been shown to be reliable and valid, evaluated in the general population on patients suffering from LBP. ${ }^{33}$ For the present study, the 2 subscales Thought Suppression Scale (TSS) and Behavioral Endurance Scale (BES) were used. The TSS consists of 4 items (eg, "Pull yourself together!"33) and measures cognitive suppression of thoughts of pain and its experiences. Internal consistency was observed to be good at Cronbach $\alpha=.80$. BES assesses 2 aspects of behavioral endurance while experiencing pain and consists of 2 subscales: Humor/Distraction Scale (5 items, eg, "I take it with a laugh"33) and Pain Persistence Scale (7 items, eg, "I keep my appointments even though I don't feel up to it"33). The participants indicated the frequency of thoughts and behavior during the past 14 days on a 7point Likert scale ranging from 0 ("never") to 6 ("always"). The internal consistency was reported as $\alpha=.83$. $^{33}$

The Beck Depression Inventory Primary Care (BDI-PC $\left.{ }^{34}\right)$ is a second important instrument in differentiating subgroups of pain behavior, in addition to the TSS and BES scales. The BDI-PC is a self-report instrument that consists of 7 items concerning sadness and loss of pleasure, suicidal thoughts or wishes, pessimism, past failure, self-dislike, and self-criticalness, all chosen from the BDIII. ${ }^{35}$ Each item ranges from 0 to 3 . One-week test-retest reliability was $r=.82 .{ }^{34}$ This screening was validated in a slightly modified form in multiple studies. ${ }^{5,33}$ If the total of the BDI-PC score was $\geq 2$, and if the TSS and BES were $<3$, then the participants were classified as showing a FAR pattern. When the BDI-PC was $\geq 2$ and TSS and/or BES were $\geq 3$, the participants were labeled as distress-endurance responders. If the BDI-PC score was $<2$ and the $\mathrm{BES} \geq 3$, a participant was classified as showing an EER pattern. If all 3 scales showed scores $<2$ (BDI-PC) or $<3$ (BES and TSS), the participants were categorized as adaptive responders.

\section{Statistical Analysis}

The means and SDs or absolute and relative frequencies of sociodemographic, pain-, and activity-related variables were calculated for the whole sample and for each, the athletes and nonathletes, separately. The Kolmogorov-Smirnov test was used to test for normality of the continuous variables. Differences between the athletes and nonathletes were inspected using Student $t$ tests for independent samples or chi-square tests. In case of a significant deviation of the normal distribution, the Mann-Whitney $U$ test was calculated.

Three 2-way analyses of variance (ANOVAs) with the factor AEM group (FAR, DER, EER, and AR) and the factor sports group (athletes and nonathletes) and the 3 features of body image (health, physical efficacy, and self-acceptance) were computed to investigate the role of AEM subgroups and being an athlete or not for body image. For pre-analyses, the Kolmogorov-Smirnov test for normality was used to test for normal distribution of the dependent variables. In the case of nonnormal distribution, however, the central limit theorem allows for ANOVAs to be conducted, as it is robust in large samples $>30 .{ }^{36}$ The Levene Test for Equality of Variances was used to inspect the homogeneity of variances of the samples. As the body image of health and the body image of selfacceptance are positively correlated with gender (men revealed higher scores than women), the body image of physical efficacy 
was negatively related to age and positively related to the current training activity per week; in a first step, the variables age and gender were included as covariates. If this procedure did not have effects on the results, only the results of the original ANOVAs were reported. In the case of main effects of the factor AEM subgroups, single contrasts were inspected using the Bonferroni post hoc test. All tests were operated by using SPSS (version 25; IBM Corp, Armonk, NY). ${ }^{37}$ The confidence intervals for all tests were at $95 \%$.

\section{Results}

A summary of the descriptive characteristics and pain parameters of athletes and nonathletes is given in Tables 1 and 2 .

The Mann-Whitney $U$ tests showed that the athletes and nonathletes displayed significant group differences with regard to age $(z=-6.193, P<.001)$, training activity (hours spent weekly; $z=-7.985, P<.001)$, and pain-related disability $(z=-2.373, P<.05)$. The athletes were younger, more active, and revealed less disability. The athletes and nonathletes displayed no significant group differences with regard to pain group (acute/subacute and chronic), pain duration in days, and BDI (see Tables 1 and 2).

With regard to the first hypothesis, concerning overall AEM subgroups and body image, the 2-way ANOVAs showed a significant main effect for all 3 body image dimensions, such as health, self-acceptance, and physical efficacy. In detail, the participants with EER and AR revealed a more positive body image compared with the participants with FAR and DER. Concerning the body image of health $\left(F_{3,238}=32.89, P<.001\right)$, the participants with EER displayed a more positive body image compared with the 2 other maladaptive pain groups $(P<.001)$ and the AR group $(P<.05)$. The participants with AR revealed a more positive body image compared with DER $(P<.001)$ and FAR $(P<.001)$. With regard to the body image of self-acceptance $\left(F_{3,238}=9.78\right.$, $P<.001)$, the Bonferroni-corrected post hoc tests revealed a more positive body image, both for EER $(P<.05)$ and AR $(P<.05)$ compared with FAR. Furthermore, EER $(P<.001)$ and AR $(P<.001)$ showed a more positive body image compared with DER. Concerning the body image of physical efficacy $\left(F_{3,238}=5.21, P<.01\right)$, the participants with EER showed a more positive body image compared with DER $(P<.01)$ and FAR $(P<.05)$. The participants with AR showed a more positive body image compared with DER $(P<.01)$ and FAR $(P<.05)($ see Table 3$)$.

The 2-way ANOVA with the factors sports group (athletes and nonathletes) and AEM groups (DER, FAR, EER, and AR) showed a significant main effect for the factor sports group in the body image of physical efficacy only $\left(F_{1,238}=22.82, P<.001\right)$, showing that the athlete group revealed a more positive body image of physical efficacy compared with the nonathlete group. Contrary to expectations, there were not more EER patterns in the group of athletes $(38 \%)$ compared with the group of nonathletes $(38.7 \%)$. Nevertheless, in both groups, the endurance patterns EER and DER (71\%) were predominant compared with AR and FAR (see Table 3).

\section{Discussion}

The objective of the present study was to examine differences in body image with respect to responses to pain (AEM subgroups) between athletes and nonathletes suffering from LBP. The results suggest that the participants with EER and AR revealed a more positive body image compared with the participants with FAR and DER, validated for all 3 body image dimensions, such as physical efficacy, self-acceptance, and health. Furthermore, athletes have a more positive body image concerning physical efficacy.

Regarding the role of AEM subgroups, our first hypothesis was supported. The athletes and nonathletes with an EER or AR to pain revealed a significantly better body image than the participants showing DER or FAR to pain. These findings were applicable for all 3 body image dimensions analyzed in the present study. These results are in line with previous research in nonathletes, showing outcomes that are more favorable in the AR and EER groups. For

Table 1 Demographics of the Sample (Athletes: $n=163$, Nonathletes: $n=75$ )

\begin{tabular}{|c|c|c|c|}
\hline & Total & Athletes & Nonathletes \\
\hline Gender & 238 & & \\
\hline Female & $106(44.5)$ & $66(40.5)$ & $40(53.3)$ \\
\hline Male & $132(55.5)$ & $97(59.5)$ & $35(46.7)$ \\
\hline Age, y & $32.02(11.71)$ & $28.69(9.60)$ & $39.34(12.63)$ \\
\hline Current training activity per week in hours & $7.65(5.43)$ & $9.28(5.61)$ & $4.12(2.68)$ \\
\hline
\end{tabular}

Note: Data are given as $\mathrm{n}(\%)$ or mean (SD).

Table 2 Pain Characteristics of the Sample

\begin{tabular}{|c|c|c|c|c|}
\hline & Total & Athletes & Nonathletes & Significance \\
\hline Pain intensity (VK-PI 0-100) & $43.85(16.45)$ & $44.31(16.04)$ & $42.84(17.39)$ & n.s. \\
\hline Pain disability (VK-DS 0-100) & $22.81(18.47)$ & $21.12(18.26)$ & $26.44(18.50)$ & $P<.05$ \\
\hline Pain duration in days & $837.49(1541.88)$ & $875.09(1582.99)$ & $756.61(1456.82)$ & n.s. \\
\hline Acute/subacute $^{\mathrm{a}}$ & $100(42.0)$ & $67(43.2)$ & $33(45.2)$ & n.s. \\
\hline Chronic $^{\mathrm{a}}$ & $128(53.8)$ & $88(56.8)$ & $40(54.8)$ & n.s. \\
\hline BDI-PC & $2.22(2.77)$ & $2.17(2.70)$ & $2.35(2.94)$ & n.s. \\
\hline
\end{tabular}

Abbreviations: BDI-PC, Beck Depression Inventory Primary Care; n.s., not significant; VK-DS, von Korff disability score; VK-PI, von Korff pain intensity. Note: Data are given as mean $(\mathrm{SD})$ or $\mathrm{n}(\%)$.

${ }^{a}$ Ten subjects did not have pain on the day of the survey, but did during the previous 7 days. 
Table 3 Avoidance-Endurance Model Pain Patterns and Means of BI

\begin{tabular}{|c|c|c|c|c|c|}
\hline & FAR $(n=31)$ & $\operatorname{DER}(n=78)$ & $\operatorname{EER}(n=91)$ & $A R(n=38)$ & Single contrasts ${ }^{a}$ \\
\hline \multicolumn{6}{|l|}{ Total } \\
\hline BI health & $22.42(3.65)$ & $21.78(4.76)$ & $26.64(3.94)$ & $28.42(4.45)$ & $\begin{array}{l}\mathrm{EER} / \mathrm{AR}>\mathrm{DER} / \mathrm{FAR} * \\
\mathrm{AR}>\mathrm{EER}\end{array}$ \\
\hline BI self-acceptance & $23.16(4.57)$ & $21.54(4.73)$ & $25.21(4.40)$ & $25.97(3.74)$ & EER/AR > DER/FAR* \\
\hline BI physical efficacy & $43.00(6.06)$ & $42.54(7.91)$ & $46.36(7.54)$ & $46.97(8.41)$ & EER/AR $>$ DER/FAR ** \\
\hline Athletes & $22(13.5)$ & $56(34.4)$ & $62(38.0)$ & $23(14.1)$ & \\
\hline BI health & $22.46(3.11)$ & $22.73(4.41)$ & $26.61(3.50)$ & $28.87(4.50)$ & EER/AR > DER/FAR* \\
\hline BI self-acceptance & $23.09(4.47)$ & $21.25(4.73)$ & $25.16(4.34)$ & $26.22(3.69)$ & EER/AR $>$ DER $*$ \\
\hline BI physical efficacy & $43.46(6.00)$ & $44.18(6.98)$ & $48.37(6.07)$ & $49.91(6.81)$ & EER/AR > DER/FAR* \\
\hline Nonathletes & $9(12.0)$ & $22(29.3)$ & $29(38.7)$ & $15(20.0)$ & \\
\hline BI health & $22.33(4.95)$ & $19.36(4.87)$ & $26.69(4.81)$ & $27.73(4.45)$ & $\begin{array}{l}\text { EER/AR }>\text { DER } * \\
\text { AR }>\text { FAR }^{*}\end{array}$ \\
\hline BI self-acceptance & $23.33(5.07)$ & $22.27(4.77)$ & $25.31(4.59)$ & $25.60(3.91)$ & n.s. \\
\hline BI physical efficacy & $41.89(6.43)$ & $38.36(8.75)$ & $42.07(8.64)$ & $42.47(8.84)$ & n.s. \\
\hline
\end{tabular}

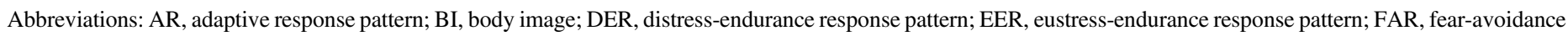
response pattern. Note: Data are given as mean $(\mathrm{SD})$ or $\mathrm{n}(\%)$.

${ }^{a}$ One-way analysis of variance with Bonferroni multiple comparison test.

$* P<.001$. ** $P<.01$.

example, Hasenbring et $\mathrm{al}^{5}$ pointed out that patients with AR and EER revealed the highest scores in positive mood. Particularly, patients with DER or FAR tend to have negative and distractive thoughts about their painful body, and these thoughts may result in chronic low back pain. ${ }^{5}$ Moreover, Cane et $\mathrm{al}^{38}$ showed that patients with low levels of avoidance and high activity reported better functioning, which could apply to the present study.

Presumably, cognitive strategies, such as recognizing pain as a signal to change something and being able to change between relaxing and performing, may be grounded in individual protective factors, like resilience, ${ }^{39,40}$ acceptance, ${ }^{41}$ and self-compassion. ${ }^{42}$ Resilience $^{43}$ and self-compassion ${ }^{44}$ were shown to be positively correlated to body image. Therefore, subgroup differences seem to be important for individualizing the therapy of LBP. The understanding of different thoughts, emotions, and behaviors of patients after the onset of LBP could help by advising patients to be more or less active. However, there is a lack of knowledge about the mechanisms promoting recovery in patients with AR and, partly, with EER. Future research should focus on these mechanisms to improve therapy options for patients with LBP. Nonetheless, this result may underpin the relevance of individual protective factors, which are working in athletes and nonathletes dependent on their socialization.

However, the results of the present study suggest that athletes and nonathletes revealed similar frequencies of EER. That may be explained by the characteristics of the present sample, in which all participants were physically active to a certain degree, as they all attended a physical exercise program.

The second hypothesis, stating that athletes reveal a more positive body image than nonathletes, was partly confirmed. As expected, athletes showed a more positive body image of physical efficacy compared with the nonathletes, which is in line with the result that athletes in the present study felt less disabled. However, the athletes and nonathletes did not differ in the body image of selfacceptance and the body image of health. These results are in accordance with previous research, though with healthy athletes. ${ }^{19,45}$ In a meta-analysis of studies in pain-free populations, Hausenblas and Symons Downs ${ }^{45}$ reported inconsistent results, insofar that athletes had fewer body image concerns, as well as a similar or even more negative body image, compared with nonathletes with respect to physical (eg, muscular physique) and psychological (eg, increased self-esteem) aspects of body image. Research comparing athletes and nonathletes with pain is lacking.

As the athletes in the present study perceived themselves, despite pain, as physically more efficient than the nonathletes, we assume that they may have greater opportunities to change training intensities or training contents than nonathletes do with regard to their physical exertion. For many athletes, training can be adapted to their current body condition because of their training experience and support from trainers and health care providers. Presumably, athletes perceive control of their training activity and, subsequently, of their pain.

Nevertheless, the athletes with LBP spent more time in training activity than nonathletes with LBP. In addition, athletes are prone to "play through the pain"24(p583) due to immanent requirements, such as funding and success. Particularly, highly competitive athletes experience many psychological benefits, which may influence the body image, ${ }^{46}$ that is, success in their sports followed by recognition. Moreover, athletes may have a more positive body image of physical efficacy because they frequently fulfill societal body ideals. ${ }^{46}$ In society, a trained body is seen to be equal to physical fitness and efficacy, which have positive connotations. In contrast, some athletes themselves tend to make comparisons with other athletes, which may result in a more negative body image. ${ }^{12}$ Furthermore, Young et $\mathrm{al}^{23}$ found 4 strategies of how athletes make pain less important for themselves: first, athletes tend to hide pain by ignoring or actively silencing the body; this is similar to Moseley's ${ }^{14}$ findings of not being aware of the painful body part and the DER pattern of the AEM; second, disrespected pain, that is, athletes evaluate pain as normal, they get used to it; third, unwelcomed pain, which describes athletes' motivation to keep their pain secret because they fear of being sorted out or criticized; and fourth, welcomed pain, for example, athletes sacrifice the body for the aspired goals. ${ }^{23}$ These strategies are potentially functional in the short term, leading to endurance behavior. Nonetheless, they seem to be highly 
dysfunctional and threatening in the long term, leading to chronic injury, negative body image, and emotional pain. ${ }^{12}$

This may explain the missing difference between athletes and nonathletes with regard to the body images of self-acceptance and health. Previous studies report that athletes may be dissatisfied with their body and, therefore, may be at higher risk for health problems such as eating disorders and worsening injuries. ${ }^{12}$ This dissatisfaction depends on the type of sport and its requirements and specific pressures of forming a perfect body. ${ }^{20,46}$ For example, male athletes may wish to lose weight (eg, ski jumpers) or gain muscle strength (eg, rugby players, boxers). Thus, some studies indicate that the higher the fat percentage in athletes, the higher the dissatisfaction with their body seems to be ${ }^{20}$ eliminating body image differences between athletes and nonathletes. Female athletes seem to struggle with the sociocultural expectations that contradict sports-related physical requirements. In the context of sports (eg, bobsleigh), a muscular body is needed, but outside the sports context, the body ideal for women is to be lean and toned. ${ }^{20}$ Furthermore, when athletes are injured or experience pain, they may perceive themselves to be vulnerable and threatened with regard to their career. The present data showed a longer pain period in the athletes compared with the nonathletes, which leads to the assumption that athletes' behavior seems to be dysfunctional in the long term and should be noted by therapists. This may have an impact on their own body image evaluation in the same way as it does for nonathletes incapacitated for work. Nevertheless, it remains unclear what has more impact on the athletes' evaluation of their body image: on the one hand athletes may perceive themselves as physically efficient despite pain and fulfilling a societal body ideal; on the other hand athletes may be dissatisfied or even anxious in concerns of their vulnerability or imperfection of their body, for example regarding their weight. However, as this present study is the first comparing LBP and body image in athletes and nonathletes, these results need to be interpreted carefully.

Considering nonathletes, there is some evidence that nonathletes with persistent pain did not differ from healthy controls regarding the physical activity level. , 17,46 This may lead to the assumption that nonathletes are less able to adjust their physical activity level when experiencing pain, as nonathletes usually do not have as much medical and training support as athletes have. As previous research has shown, nonathletic patients with persistent pain had a more negative body image in the 3 examined dimension (physical efficacy, self-acceptance, and health), compared with healthy controls ${ }^{17}$; this inability of adaptation may be a possible explanation for the present results.

With regard to the impact of physical activity in general, there is evidence that physical activity, particularly exercise intervention, has positive effects on body image. ${ }^{18}$ This might be another explanation for the nonexistent difference between athletes and nonathletes concerning self-acceptance and health. At the least, the nonathletes with chronic low back pain in the present study and in general may benefit from instructed exercise treatment. Furthermore, as physical activity was shown to be associated with positive effects on pain, ${ }^{47}$ the physical activity itself may account for the low to moderate level of pain intensity and pain-related disability in both groups. Exercise may improve the pain characteristics and, especially, the body image of health in nonathletes with persistent pain. However, research has shown that the dose of physical activity is related to pain. Low and high levels of physical activity are associated with higher pain grades. ${ }^{48}$ Otherwise, a positive body image itself may provide a reason for undertaking training activity, as having a good bodily shape presents a strong motivator. ${ }^{18}$ However, there is still uncertainty regarding the mechanisms through which training activity improves body image. Because LBP may be influenced both by training activity and body image (and vice versa), examining this triad in more detail seems to be worthwhile in future research.

\section{Limitations}

The present study has some limitations. A first limitation is that some nonathletes revealed high training frequencies, coming close to athletes' frequencies, or rather, may have been an athlete in the past, which was not assessed. As there is evidence suggesting that physical activity may positively influence body image, the present sample may have had higher scores in body image. Therefore, including only people from a physiotherapeutic setting may have caused a selection effect with regard to body image. Furthermore, having been an athlete and therefore possibly having developed some kind of athletic identity ${ }^{49}$ may have affected the body image positively as well. A second limitation is that, due to the crosssectional nature of the study, cause and effect regarding body image, physical activity and pain behavior cannot be interpreted. As there is evidence for a time dependence of these mechanisms (eg, pain management in athletes differs depending on pain duration $^{50}$ ), longitudinal designs will be needed in future research. Finally, the athletes in the present study were younger than nonathletes. Although the variable age as covariate did not influence the results, there is evidence that age and body image are related insofar that satisfaction with one's own body may vary through one's lifetime (see Grogan ${ }^{51}$ and Tiggemann ${ }^{52}$ ). This applies to gender, as well. For instance, there is evidence that men reveal more positive body image when they are younger compared with women (see Ricciardelli and $\mathrm{McCabe}^{53}$ ). Finally, in addition to these aspects, future studies should (1) distinguish between disciplines, for example, endurance, strength, and/or individual and team sports, as the incidence for LBP as well as body image differs, and (2) include healthy participants. In summary, our results have to be evaluated critically.

\section{Conclusion}

The present study is the first step to bring the important dimensions of body image and pain responses together. The results suggest that body image and dysfunctional pain responses are related, and furthermore, that more individual aspects should be considered regarding the occurrence and maintenance of LBP. In particular, participants showing a pattern of pain response tainted with a positive mood revealed a more positive body image, which is valid for all 3 dimensions, such as physical efficacy, self-acceptance, and health. This needs to be explored in more detail in the future. Furthermore, the athletes revealed a more positive body image of physical efficacy compared with the nonathletes. This seems to be relevant for therapy approaches for nonathletes in the sense that focusing on even a small progress and, therefore, feeling more flexible and physically efficient could help the patients with regard to acceptance. Therefore, body image and pain responses are 2 relevant points. Thus, future research should focus on these aspects to improve therapy options.

\section{Acknowledgments}

This work was supported by the German Federal Institute of Sport Science within the National Research Network for Medicine in Spine Exercise 
(MiSpEx) under grant number IIA1-080102B/11-14. All procedures performed in studies involving human participants were in accordance with the ethical standards of the institutional and/or national research committee and with the 1964 Helsinki Declaration and its later amendments or comparable ethical standards. There was no involvement in any aspect of the study. The authors declare that they have no conflict of interest.

\section{References}

1. Hasenbring MI, Levenig C, Hallner D, et al. Psychosoziale Risikofaktoren für chronischen Rückenschmerz in der Allgemeingesellschaft und im Leistungssport. Von der Modellbildung zum klinischen Screening-ein Review aus dem MiSpEx-Netzwerk [Psychosocial risk factors for chronic back pain in the general population and in competitive sports. From theory to clinical screening-a review from the MiSpEx network]. Schmerz. 2018;32:259-273. PubMed ID: 29946960 doi:10.1007/s00482-018-0307-5

2. Fett D, Trompeter K, Platen P. Back pain in elite sports: a crosssectional study in 1114 athletes. PLoS One. 2017;12:e180130. PubMed ID: 28662110 doi:10.1371/journal.pone.0180130

3. Linton SJ. A review of psychological risk factors in back and neck pain. Spine. 2000;25:1148-1156. PubMed ID: 10788861 doi:10. 1097/00007632-200005010-00017

4. Heidari J, Mierswa T, Kleinert J, et al. Parameters of LBP chronicity among athletes: associations with physical and mental stress. Phys Ther Sport. 2016;21:31-37. PubMed ID: 27428532 doi:10.1016/j. ptsp.2016.03.003

5. Hasenbring MI, Hallner D, Klasen B, Streitlein-Böhme I, Willburger $\mathrm{R}$, Rusche H. Pain-related versus endurance in primary care patients with subacute back pain: psychological characteristics and outcomes at a 6-month follow-up. Pain. 2012;153:211-217. PubMed ID: 22093816 doi:10.1016/j.pain.2011.10.019

6. Hasenbring M, Verbunt JA. Fear-avoidance and endurance-related responses to pain: new models of behavior and their consequences for clinical practice. Clin J Pain. 2010;26:747-753. PubMed ID: 20664333 doi:10.1097/AJP.0b013e3181e104f2

7. Hasenbring MI, Chehadi O, Titze C, Kreddig N. Fear and anxiety in the transition from acute to chronic pain: there is evidence for endurance besides avoidance. Pain Manag. 2014;4:363-374. PubMed ID: 25350076 doi:10.2217/pmt.14.36

8. Plaas H, Sudhaus S, Willburger R, Hasenbring MI. Physical activity and low back pain: the role of subgroups based on the avoidanceendurance model. Disabil Rehabil. 2014;36:749-755. PubMed ID: 23865908 doi:10.3109/09638288.2013.814723

9. Vlaeyen JW, Linton SJ. Fear-avoidance and its consequences in chronic musculoskeletal pain: a state of the art. Pain. 2000; 85:317-332. PubMed ID: 10781906 doi:10.1016/S0304-3959(99) 00242-0

10. Konietzny K, Chehadi O, Levenig C, et al. Depression and suicidal ideation in high-performance athletes suffering from low back pain: the role of stress and pain-related thought suppression. Eur J Pain. 2019;23:1196-1208. PubMed ID: 30821880 doi:10.1002/ejp.1387

11. Heidari J, Belz J, Hasenbring MI, Kleinert J, Levenig C, Kellmann M. Examining the presence of back pain in competitive athletes: a focus on stress and recovery. J Sport Rehabil. 2019;28:188-195. doi:10. 1123/jsr.2017-0235

12. McEwen K, Young K. Ballet and pain: reflections on a risk-dance culture. Qual Res Sport Exerc Health. 2011;3:152-173. doi:10.1080/ 2159676X.2011.572181
13. Gajsar H, Titze C, Levenig C, et al. Psychological pain responses in athletes and non-athletes with low back pain: avoidance and endurance matter. Eur J Pain. 2019;23:1649-1662. PubMed ID: 31220382 doi:10.1002/ejp.1442

14. Moseley GL. I can't find it! Distorted body image and tactile dysfunction in patients with chronic back pain. Pain. 2008; 140:239-243. PubMed ID: 18786763 doi:10.1016/j.pain.2008. 08.001

15. Deusinger IM. Frankfurter Körperkonzeptskalen [Frankfurt Body Concept Scales]. Göttingen, Germany: Hogrefe; 1998.

16. Levenig CG, Hasenbring MI, Kleinert J, Kellmann M. Körperbild und Rückenschmerz [Body image and low back pain]. Schmerz. 2016;30:437-443. PubMed ID: 27333766 doi:10.1007/s00482-0160122-9

17. Levenig CG, Kellmann M, Kleinert J, Belz J, Hesselmann T, Hasenbring MI. Body image is more negative in patients with chronic low back pain than in patients with subacute low back pain and healthy controls. Scand J Pain. 2019;19:147-156. PubMed ID: 30205663 doi:10.1515/sjpain-2018-0104

18. Petrie TA, Greenleaf C. Body image and athleticism. In: Cash TF, Smolak L, eds. Body Image: A Handbook of Science, Practice, and Prevention. 2nd ed. New York, NY: Guilford Press; 2012:206-213.

19. Hausenblas HA, Fallon EA. Exercise and body image: a metaanalysis. Psychol Health. 2006;21:33-47. doi:10.1080/147683205 00105270

20. Gibson C, Hindle C, McLay-Cooke R, et al. Body image amongst elite rugby union players. J Strength Cond Res. 2019;33:2217-2222. doi:10.1519/JSC.0000000000002312

21. Hausenblas HA, McNally KD. Eating disorder prevalence and symptoms for track and field athletes and nonathletes. J Appl Sport Psychol. 2004;16:274-286. doi:10.1080/10413200490485630

22. Bettle N, Bettle $\mathrm{O}$, Neumärker U, Neumärker K. Body image and self-esteem in adolescent ballet dancers. Percept Mot Skill. 2001; 93:297-309. doi:10.2466/pms.2001.93.1.297

23. Young K, White P, McTeer W. Body talk: male athletes reflect on sport, injury, and pain. Sociol Sport J. 1994;11:175-194. doi:10. 1123/ssj.11.2.175

24. Deroche T, Woodman T, Stephan Y, Brewer BW, Le Scanff C. Athletes' inclination to play through pain: a coping perspective. Anxiety Stress Coping. 2011;24:579-587. PubMed ID: 21328106 doi:10.1080/10615806.2011.552717

25. Diehl K, Mayer J, Thiel A, Zipfel S, Schneider S. „Playing hurt“: der Umgang jugendlicher Leistungssportler mit Gelenkschmerzen ["Playing hurt": dealing with joint pain in adolescent elite athletes]. Schmerz. 2019;33:49-56. PubMed ID: 29294211 doi:10.1007/ s00482-017-0263-5

26. Newell R. Body-image disturbance: cognitive behavioural formulation and intervention. $J$ Adv Nurs. 1991;16:1400-1405. PubMed ID: 1791249 doi:10.1111/j.1365-2648.1991.tb01586.x

27. Sharma P, Sandhu JS, Shenoy S. Variation in the response to pain between athletes and non-athletes. Ibnosina J Med Biomed Sci. 2011;3:165-171. doi:10.4103/1947-489X.210889

28. Heidari J, Mierswa T, Hasenbring M, et al. Low back pain in athletes and non-athletes: a group comparison of basic pain parameters and impact on sports activity. Sport Sci Health . 2016;12:297-306. doi:10. 1007/s11332-016-0288-7

29. Araújo CG, Scharhag J. Athlete: a working definition for medical and health sciences research. Scand J Med Sci Sports. 2016;26:4-7. doi:10.1111/sms. 12632

30. Klasen BW, Hallner D, Schaub C, Willburger R, Hasenbring M. Validation and reliability of the German version of the Chronic Pain 
Grade questionnaire in primary care back pain patients. Psychosoc Med. 2004;1:Doc07. PubMed ID: 19742049

31. Von Korff M, Ormel J, Keefe FJ, Dworkin SF. Grading the severity of pain. Pain. 1992;50:133-149. PubMed ID: 1408309 doi:10.1016/ 0304-3959(92)90154-4

32. Balagué F, Mannion AF, Pellisé F, Cedrashi C. Non-specific low back pain. Lancet. 2012;379:482-491. doi:10.1016/S0140-6736(11) 60610-7

33. Hasenbring MI, Hallner D, Rusu AC. Fear-avoidance- and endurance-related responses to pain: development and validation of the Avoidance-Endurance-Questionnaire (AEQ). Eur J Pain. 2009;13:620-628. PubMed ID: 19101182 doi:10.1016/j.ejpain. 2008.11.001

34. Beck AT, Guth D, Steer RA, Ball R. Screening for major depression disorders in medical inpatients with the Beck Depression Inventory for Primary Care. Behav Res Ther. 1997;35:785-791. PubMed ID: 9256522 doi:10.1016/S0005-7967(97)00025-9

35. Beck AT, Steer RA, Brown GL. Manual for the Beck Depression Inventory-II. San Antonio, TX: Psychological Corporation; 1996.

36. Field A. Discovering Statistics Using IBM SPSS Statistics. Los Angeles, CA: Sage; 2015.

37. IBM Corporation. IBM SPSS Statistics for Windows. Armonk, NY: IBM Corporation; 2015.

38. Cane D, Nielson WR, Mazmanian D. Patterns of pain-related activity: replicability, treatment-related changes, and relationship to functioning. Pain. 2018;159:2522-2529. PubMed ID: 30074594 doi:10. 1097/j.pain.0000000000001357

39. Jegan NRA, Brugger M, Viniol A, et al. Psychological risk and protective factors for disability in chronic low back pain-a longitudinal analysis in primary care. BMC Musculoskelet Disord. 2017;18:114. PubMed ID: 28320375 doi:10.1186/s12891-017-1482-8

40. Karoly P, Ruehlman LS. Psychological "resilience" and its correlates in chronic pain: findings from a national community sample. Pain. 2006;123:90-97. PubMed ID: 16563626 doi:10.1016/j.pain.2006. 02.014

41. Costa J, Pinto-Gouveia J. Acceptance of pain, self-compassion and psychopathology: using the Chronic Pain Acceptance Questionnaire to identify patients' subgroups. Clin Psychol Psychother. 2011; 18:292-302. PubMed ID: 20806418 doi:10.1002/cpp.718

42. Neff KD. The development and validation of a scale to measure self-compassion. Self Ident. 2003;2:223-250. doi:10.1080/152988 60309027
43. Izydorczyk B, Kwapniewska A, Lizinczyk S, Sitnik-Warchulska K. Psychological resilience as a protective factor for the body image in pots-mastectomy women with breast cancer. Int J Environ Res Public Health. 2018;15:1181. doi:10.3390/ijerph15061181

44. Braun TD, Park CL, Gorin A. Self-compassion, body image, and disordered eating: a review of the literature. Body Image. 2016; 17:117-131. PubMed ID: 27038782 doi:10.1016/j.bodyim.2016. 03.003

45. Hausenblas HA, Symons Downs D. Comparison of body image between athletes and nonathletes: a meta-analytic review. $J$ Appl Sport Psychol. 2001;13:323-339. doi:10.1080/104132001753 144437

46. Bousema EJ, Verbunt J, Seelen HAM, Vlaeyen JWS, Knottnerus JA. Disuse and physical deconditioning in the first year after onset of back pain. Pain. 2007;130:279-286. PubMed ID: 17467902 doi:10.1016/j. pain. 2007.03 .024

47. Cote JN, Hoeger Bement MK. Update on the relation between pain and movement: consequences for clinical practice. Clin J Pain. 2010;26:754-762. PubMed ID: 20664335 doi:10.1097/AJP. 0b013e3181e 0174f

48. Heneweer H, Picavet HSJ, Staes F, Kiers H, Vanhees L. Physical fitness, rather than self-reported physical activities, is more strongly associated with low back pain: evidence from a working population. Eur Spine J. 2012;21:1265-1272. PubMed ID: 22134487 doi:10. 1007/s00586-011-2097-7

49. Jessiman-Perreault G, Godley J. Playing through the pain: a university-based study of sports injury. Adv Phys Educ. 2016; 6:178-194. doi:10.4236/ape.2016.63020

50. Kleinert J. Dimensionen adaptiver Schmerzbewältigung im Sport [Dimensions of adaptive pain management in sports]. Psychol Sport. 2000;7:3-14.

51. Grogan S. Body image development in adulthood. In: Cash TF, Smolak L, eds. Body Image: A Handbook of Science, Practice, and Prevention. 2nd ed. New York, NY: Guilford Press; 2012: 93-100.

52. Tiggemann M. Considerations of positive body image across various social identities and special populations. Body Image. 2015;14:168176. PubMed ID: 25865460 doi:10.1016/j.bodyim.2015.03.002

53. Ricciardelli LA, McCabe MP. Body image development in adolescent boys. In: Cash TF, Smolak L, eds. Body Image: A Handbook of Science, Practice, and Prevention. 2nd ed. New York, NY: Guilford Press; 2012:85-92. 